\title{
Soil Moisture Monitoring System based on Wireless Sensor Network
}

\author{
Lukai Lin ${ }^{1, a}$, Weiying Gu ${ }^{1, b}$, Xiangshun $\mathrm{Li}^{1, \mathrm{c}}$ and Guangjin Shen ${ }^{2, \mathrm{~d}}$ \\ 'WuHan City, HuBei Province, China \\ ${ }^{2}$ BaoDing City, HeBei Province, China \\ alinllk@whut.edu.cn, b1337263317@qq.com, c53290323@qq.com, d987730326@qq.com
}

\begin{abstract}
Keywords: ZigBee, Wireless Sensor Network (WSN), Soil moisture monitoring, GPRS
Abstract: Aiming at the current demand of water-saving agriculture, improving the utilization rate of agricultural irrigation water and further promoting fine irrigation, a soil moisture monitoring system based on wireless sensor network (WSN) was designed., The system combines the advantages of ZigBee's low cost, low power consumption and high reliability and the advantages of GPRS's capability of remote transmission of data. In addition, powered by solar energy, the system is easier to maintain and more reliable in use. The preliminary test has validated the rationality and practicability of the system, which is helpful to popularize water-saving agriculture and increase crop yield.
\end{abstract}

\section{Introduction}

The total amount of water resources in China ranks sixth in the world, nevertheless, the per capita water resources accounted for only $1 / 4$ of the world's per capita consumption. As a large agricultural country, Chinese long-term practice of large area irrigation method has resulted in a serious waste of water resources. Therefore, the rational use of agricultural water resources and vigorously promoting water-saving agriculture is particularly important. Soil water is the most fundamental substance for the plants, so soil moisture has great influence upon crop growth, cultivation, planting quality and soil temperature. The soil moisture is also referred to as soil humidity indicating the water content in the soil and water supply for the farm plants.[1] However, it is extremely difficult to obtain the soil moisture information in real time because of the particularity of agricultural production environment and production process. The traditional detection methods of soil moisture with small monitoring range, low degree of automation and inaccurate monitoring have great limitation in practical application.

To solve the problem of soil moisture detection, the WSN gateway node based on TI's CC2530 chip with low power consumption design, integrated MCU and RF circuit was designed. Its function is to realize the collection of the whole network monitoring data, and to regularly send the soil moisture data to the mobile phone by GPRS. [2] The use of WSN for monitoring a variety of site conditions for on-farm decision making in Precision Agriculture (PA) is becoming feasible and cost -effective. With the recent advent of low cost, low powered remote sensor nodes, a significant increase in the extent of coverage area and the number of sensor parameters measured at real time could be observed. [3] Using Zigbee to build a wireless sensor network with low power consumption, low cost and high reliability of the soil moisture monitoring system, in order to resolve the current soil moisture monitoring of low degree of automation and the foreign technical problem of high cost, has a positive role in the promotion of water-saving agriculture. The structure is arranged as follows: (2) System design: overview of the overall system of the program and implementation methods. (3) Hardware components: details of the hardware structure of the system and the link between the hardware. (4) Software algorithm: research on how to minimize energy consumption in the case of efficient operation of the system. (5) Physical verification: the hardware and software are used to realize the system in reality. (6) Conclusion: the corresponding conclusions drawn through the research of this system. 


\section{System Design}

2.1 General Structure of Wireless Soil Moisture Monitoring System. The wireless sensor network consists of the coordinator, router, and terminal node. The coordinator is responsible for the initial communication channel, initializes the network configuration and accepts the child nodes to join the network. It also has the full functionality of the router. Compared to the coordinator and router, which are collectively referred to as full function device (FFD) [4], terminal nodes can only find and connect routers. The WSN-based soil moisture monitoring system distributes a large number of nodes in various areas of farmland. These nodes use Zigbee technology to form a regional wireless sensor network, which includes a coordinator and several routers and terminal nodes. The coordinator implements the functions of the entire Zigbee protocol network, data aggregation processing and GPRS communication, additionally, maintains the whole network. It is a hub for wireless sensor networks to interact with the outside world. Through it, users can remotely read real-time soil moisture and analyze the data. The routing node also collects the soil moisture around it while the data routing is implemented. And the terminal node is at the end of the whole wireless sensor network, responsible for sending the measured data after joining the network.

WSNs allow the coverage of wide geographical areas. The range of the area depends on several factors such as the number of nodes, the way that have been placed and the range of the wireless units.[5] In practice, due to the limited transmission distance of the sensor nodes, the farmland needs to be divided into an area with equal area of blocks. As shown in Fig. 1, in order to minimize the cost with the minimum number of sensors to cover the maximum farmland area, the system uses the honeycomb distribution method to divide the farmland into one equal area hexagon. The end node is placed at the vertex of the hexagon, the routing node is placed in the center of the regular hexagon, and the coordinating node is placed on the farm side to receive the data from the routing node and transmit data remotely.

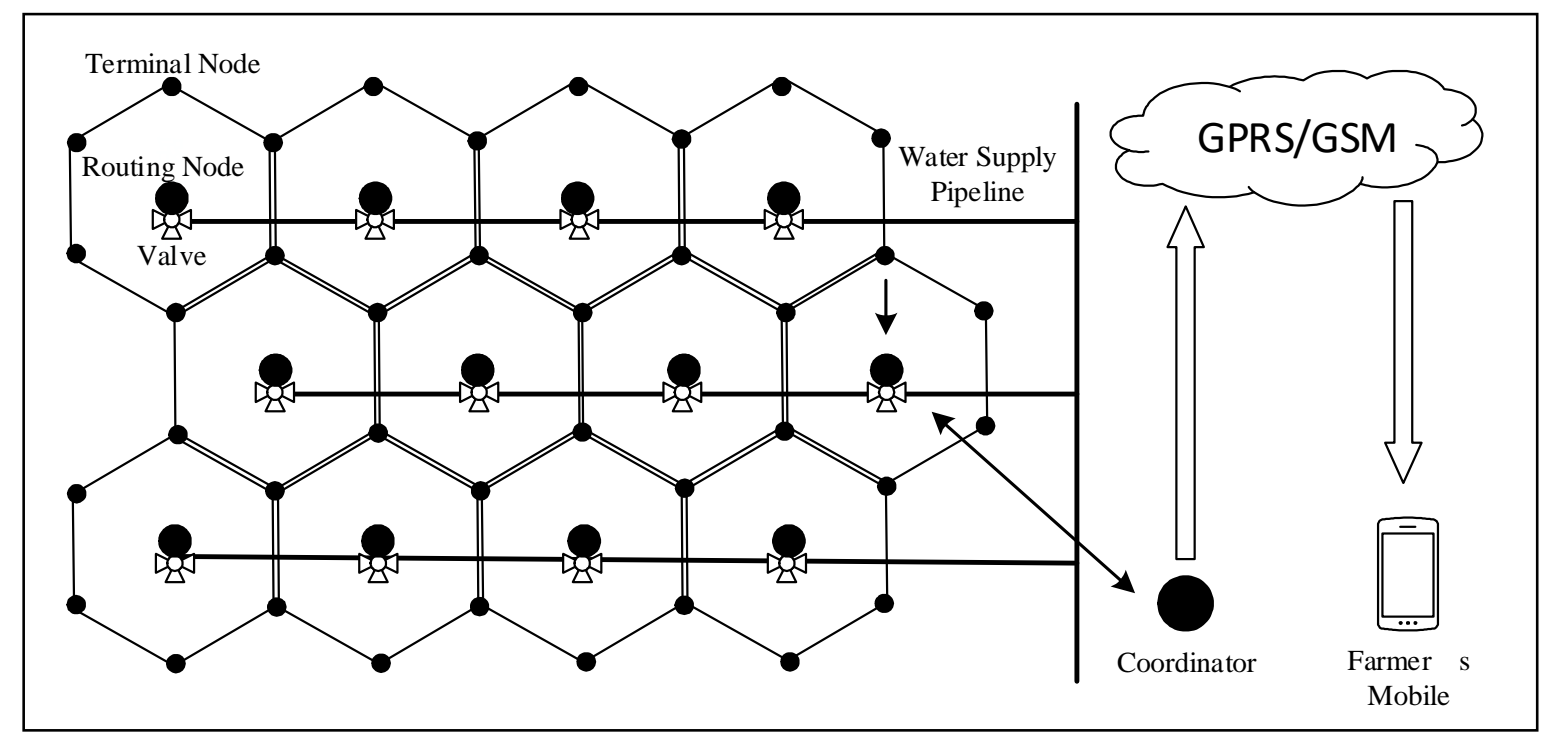

Fig. 1 Schematic diagram of soil moisture monitoring system

2.2 Working Principle. As shown in Fig. 2, the system is first started by the coordinator, and then enters the networking process. In the networking process, according to the Zigbee protocol, a series of sessions are performed between the layers of the coordinator to complete the configuration of the new network until the network is established successfully. After that, the routing node begins to join the network, routers and coordinator layers of the agreement to complete their own configuration through a series of sessions, and then each node in the network can communicate [6]. At this time, the sensor terminal node carries on the data collection work, and transmits the data packet to the adjacent routing node. The routing node collects the data and also receives the data packets from the terminal node, and then sends the data to the coordinator. The routing node itself also collects the data and 
sends all the data together to the coordinator. Then the coordinator transmits the data to the farmer's mobile phone through GPRS / GSM, so as to achieve the purpose of real-time monitoring.

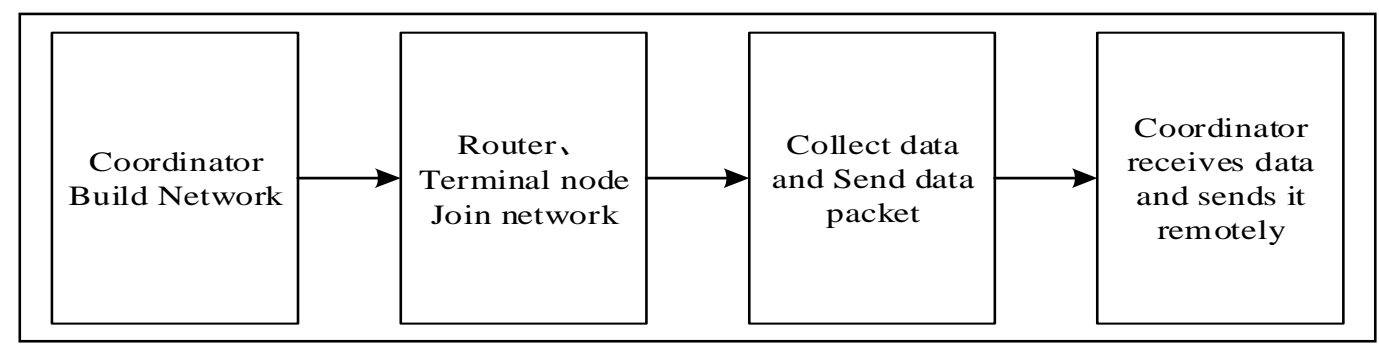

Fig. 2 Schematic diagram of the system

\section{Hardware Component}

3.1 Wireless Transceiver Module. As shown in Fig. 3, the design of the data transmission in this system is mainly completed by Texas Instrument'sCC2530 and RF power amplifier module CC2591. The CC2530 is a real system-on-a-chip solution for 2.4GHz IEEE 802.15.4, Zigbee and RF4CE applications. It combines the outstanding performance of the leading RF transceivers and the industry's enhanced 8051CPU, which can build strong networks with very low total cost. [7]CC2591 is a front-end RF which used in $2.4 \mathrm{GHz}$ band with low cost and high performance. It integrates power amplifier, low noise amplifier, balanced converter and RF matching network, and its data transmission range can be expanded to 600 meters when it works with CC2530.

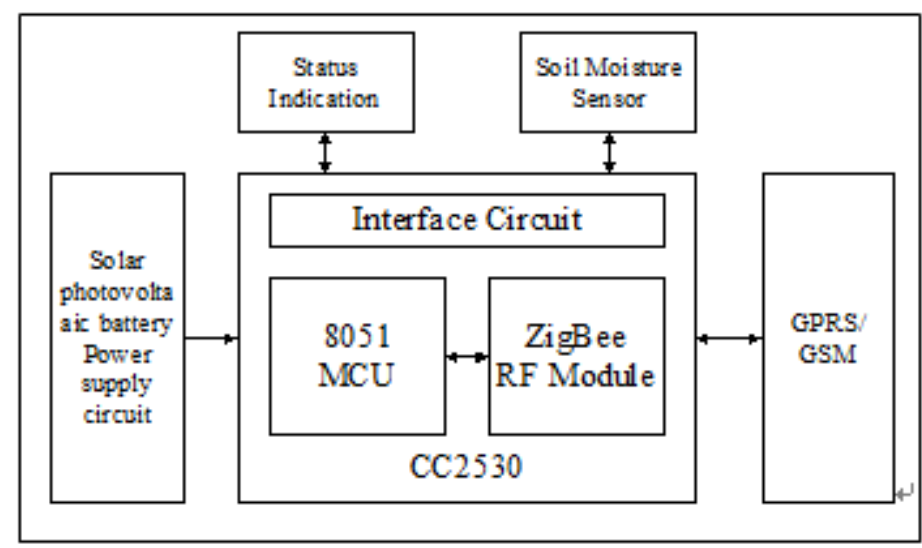

Fig. 3 node structure

3.2 Data Acquisition Module. The soil moisture sensor uses a dielectric sensor based on frequency domain reflectometry (FDR) to measure soil moisture content. Based on the principle of electromagnetic pulse, FDR is used to measure the dielectric constant of soil according to the velocity of electromagnetic wave propagation in soil, so that the soil volumetric water content can be calculated. Using a pair of electrodes as a capacitor, in where the soil acts as a dielectric. The capacitor and an oscillator form a tuning circuit that transfers the frequency signal to the dielectric sensor, and then uses frequency sweep method to detect the resonant frequency. [8]As shown in Fig. 4 , the probe is inserted into the soil, different soil water content corresponding to different impedance, which affects the output voltage and indirectly reflect the corresponding soil moisture.

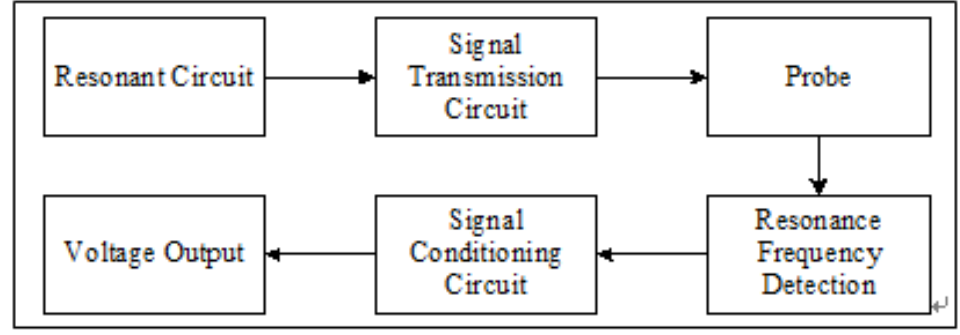

Fig. 4 principle of soil moisture sensor 
3.3 Power Module. The design of the power supply uses a rechargeable lithium battery with solar power supply, solar cell board charge the lithium battery during the day and the node is charged by the lithium battery alone at night. Solar charging management circuit is mainly composed of multi type battery charging management integrated circuit ZS6093. The module is a PWM buck mode charge management IC, with the maximum power point tracking function of solar cells. The constant current and constant voltage charging mode can be used to manage the charging of single or multi-section lithium battery completely. In addition, the small package size, few peripheral devices and the simplicity of usage are its great advantages when it works in the wireless sensor networks.

3.4 GPRS Communication Module. In order to send the data collected by the sensor network in real time, the design adopts GPRS / GSM, because its wide coverage and long communication distance are suitable for the communications between wireless sensor networks and external. Because of its flow billing, small amount of data in soil moisture monitoring system and low real-time requirements, the system is very suitable for the use of this method for data transmission.

This design uses the SIM900A module produced by SIMCom company. The chip uses a power saving technology designed to minimize power consumption in SLEEP mode to only 1.0mA, which is suitable for most of the low power requirements. Chip embedded TCP/IP protocol, the SIM900A uses the standard AT command to write to achieve the TCP connection with the central server, so that the data collected by ZigBee network transfer out [2]. This module uses SMT package, compact structure, easy production and processing; and based on the ARM926EJS architecture. Its performance is very strong, which can be widely used in wireless sensor network, vehicle tracking, fleet management, smart meter etc.

\section{Software Algorithm}

4.1 ZigBee Protocol Profile. Wireless sensor network node communicate data between each other through wireless network protocol (including MAC, routing layer, network layer, application layer), nevertheless, the traditional wireless protocols is difficult to meet the low cost, low power consumption and high reliability requirements. In this case, the Zigbee protocol came into being. Zigbee defines a range of communication protocols required for wireless communication at short-range, low-rate transmission rates, enabling a large number of sensors to communicate with each other with minimal energy, so it has high communication efficiency. The design develops based on TI's Z-Stack protocol stack. As a core software platform for developing 802.15.4 / ZigBee technology, users only need to follow the IEEE802.15.4 standard for a series of initialization operations and through the polling of the operating system configuration and high-level call to complete the corresponding application design, to develop out of the corresponding function of the wireless sensor network. [9].

4.2 Acquisition Node Programming. The main function of the terminal node in the wireless sensor network is to collect the data. After the power-on initialization is complete, the terminal node sends the network request to the coordinator. Received the request, the sensor node then sends the connection request to the coordinator. If the request response is correct, the network entry succeeds; otherwise fails. After the network is successful, the coordinator assigns the node a unique ID, and the MAC address of the sensor node is associated with the ID registration [10]. The working flow chart of the sensor node is shown in Fig. 5. After the network, the sensor node collects the soil moisture once every hour and sends out the data packet, and enters the sleep mode in the idle time. Only interrupt, timer and wireless transceiver module, so as to achieve the purpose of low power consumption. 


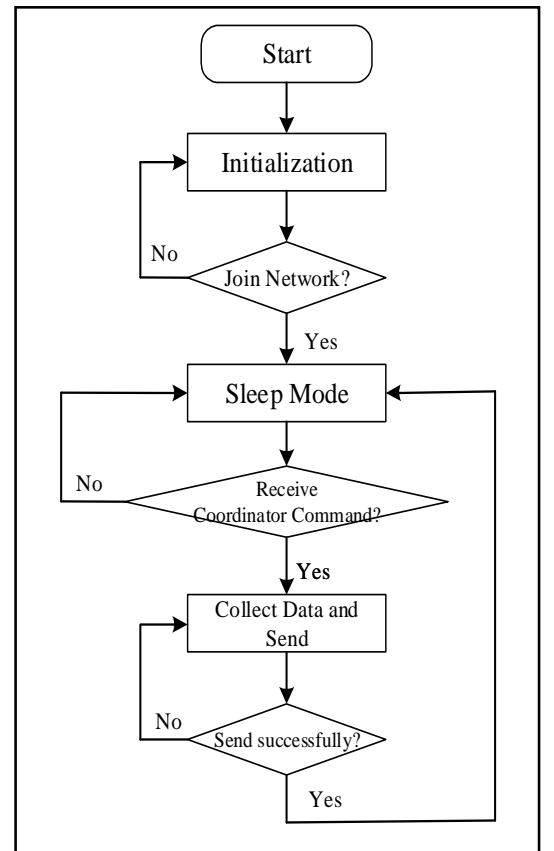

Fig. 5 flow chart of acquisition node

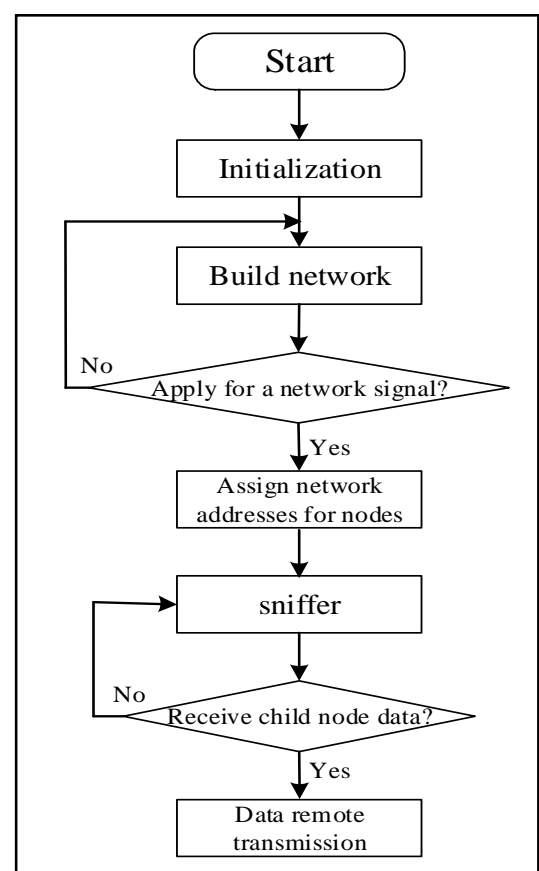

Fig. 6 flow chart of coordinator

4.3 Coordinator Node Programming. As the center of the entire sensor network, the coordinator node is primarily responsible for networking and wirelessly transmitting the data of the network remotely. Initialized the power of the coordinator node, the coordinator node starts to set up the network and then enters the network listening state. After receiving the network node request, the node is assigned the address and sends the network confirmation information, and then the connection is established [11]. The Z-Stack in the sensor network is in the form of an operating system. By constantly querying whether an event occurs, once the packet sent from the child node is received, the coordinator sends the corresponding AT command to the SIM900A through the serial port, so that the data can be transmitted over GPRS remotely. Coordinator work flow chart shown in Fig. 6.

\section{Physical Verification}

In order to verify the reliability and stability of the design, the following tests were carried out: a piece of farmland was selected, in which six terminal nodes were placed in a honeycomb shape and each end node was fitted with a soil moisture sensor to measure the data. The soil moisture data in the test are shown in Table 1 and Table 2. From the experimental results, the method can correctly receive the collected data with strong reliability.

Table 1 data acquisition at different time of the same node

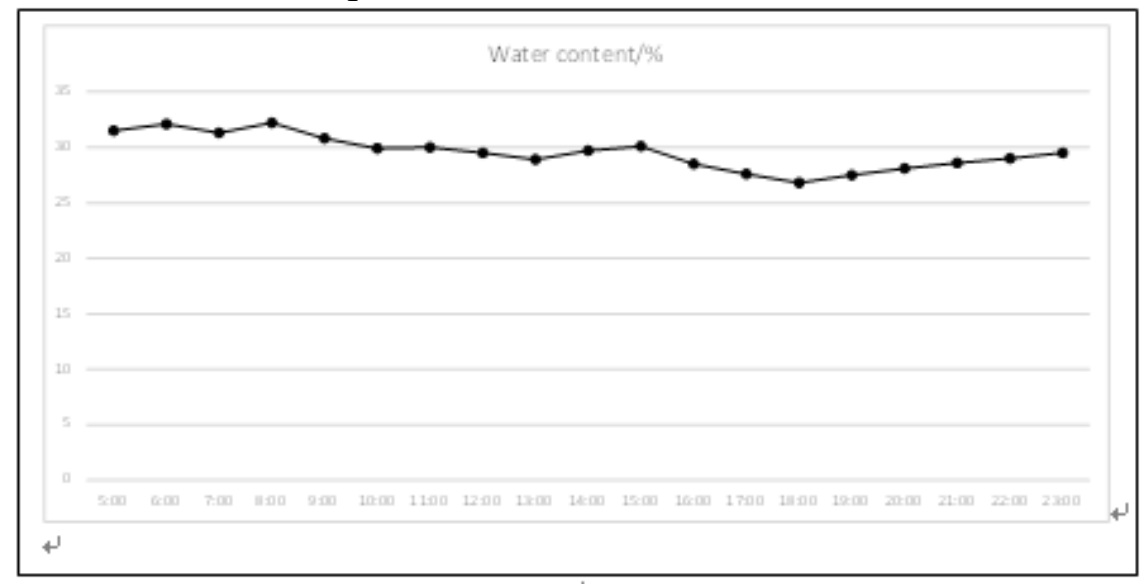


Table 2 data values of different nodes at the same time

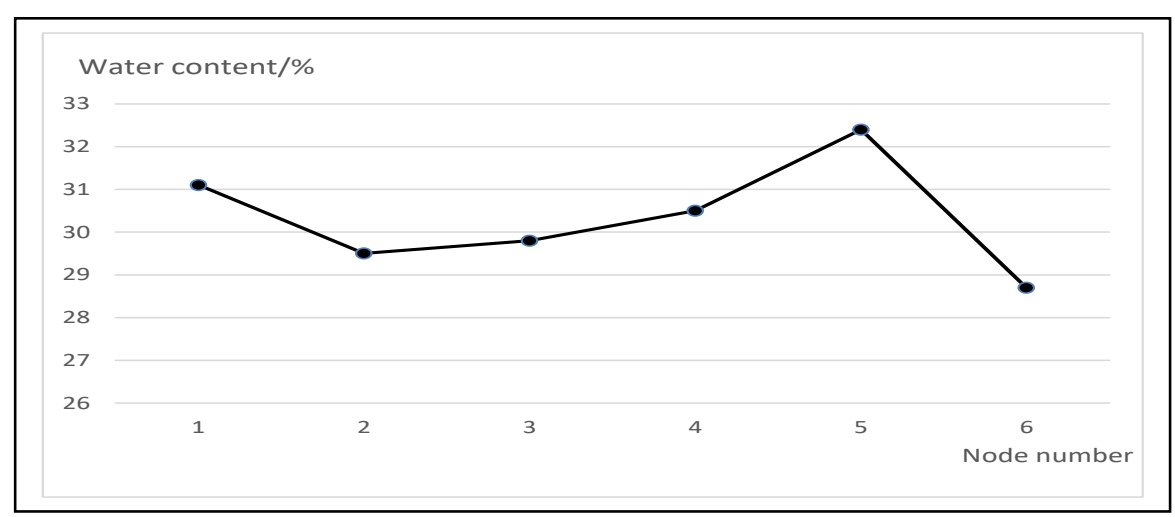

\section{Conclusion}

The system combines the ZigBee wireless sensor network with GPRS / GSM communication to apply the soil moisture monitoring system based on the wireless sensor network. It uses the CC2530 and SIM900A to build the sensor network and uses solar energy to power the network nodes. According to the field test results, the designed wireless sensor network has high reliability and good stability. And its low power consumption, low cost, easy installation and maintenance are suitable for various terrain farmland environment, also meet the needs of farmland soil moisture remote monitoring, which has great practical significance.

\section{References}

[1] S. S. Wan, Fundamentals of Soil Physics, National Institute for Compilation and Translation, 2001, pp. 136-145.

[2] Chen Ketao, Zhang Haihui,Zhang Yongmeng, et al. Design of CC2530 based gateway node for wireless sensor network[J]. Journal of Northwest A\&F University(Nat. Sci. Ed.), 2014, 42(5):183-188. (in Chinese with English abstract)J. Clerk Maxwell, A Treatise on Electricity and Magnetism, 3rd ed., vol. 2. Oxford: Clarendon, 1892, pp.68-73.

[3] Jayashree L S, Yamini V K, Priya R M. A Communication Efficient Framework for Soil Moisture Monitoring using Wireless Sensor Networks[J]. International Journal of Computer Applications, 2011, 1(16):16-23.

[4] Li Wenzhong, Duan Chaoyu. Zigbee2006 wireless network and wireless positioning[M]. Beihang University Press, 2008. (in Chinese).

[5] Giannopoulos N, Goumopoulos C, Kameas A. Design Guidelines for Building a Wireless Sensor Network for Environmental Monitoring[C]// Informatics, 2009. Pci '09. Panhellenic Conference on. IEEE, 2009:148-152.

[6] Hu Peijin, Jiang Ting, Zhao Yandong. Monitoring system of soil water content based on zigbee wireless sensor network[J].Transactions of the CSAE, 2011,27(4):230-234. (in Chinese with English abstract).

[7] CC2530 PRELIMINARY DataSheet Texas Instruments, 2011.

[8] Wang Jun, He Zhitao. Development of soil moisture measurement circuit[J]. Water Saving Irrigation, 2008(7):24-26. (in Chinese).

[9] Bao Changchun, Shi Ruizhen, Ma Yuquan, et al. Design and realization of measuring and controlling system based on ZigBee technology in agricultural facilities[J]. Transations os the CASE, 2007, 23(8): 160-164.(in Chinese with English abstract). 
[10] Wang Hui, Dong Xueren, Yang Xiaowei, et al. Design of Greenhouse Wireless Monitoring System Based on ZigBee[J]. Instrumentation•Analysis•Monitoring, 2012(2):30-33. (in Chinese).

[11] Jia Kejin, Wang Wenzhen, Du Taihang, et,al. Soil moisture monitoring system based on ZigBee wireless sensor network[J]. Water Saving Irrigation, 2014(3):69-71. (in Chinese). 